\title{
Iliohypogastric Nerve Block for Chronic Pelvic Pain Treatment: Ultrasound-Guide Technique Description and Case Series
}

Presented at 1st International Online BioMedical Conference

Marta Linares Gago ${ }^{1}$, Gonzalo Cano Plasencia ${ }^{1}$, Inmaculada Díaz-Cano Carmona ${ }^{1}$, Jorge Rodríguez García ${ }^{1}$, Luis Eduardo Alarcón Mora ${ }^{1}$

\section{Abstract}

Background: Chronic pelvic pain is common and often difficult to alleviate. The aim of this study is to describe the technique of the lliohypogastric Nerve (IPN) block with ultrasound-guide (UG) and show its utility and safety in a Physical Medicine and Rehabilitation Unit.

Aim and Objetives: To Todescribe the technique of the lliohyogastric Nerve (IPN) block with ultrasound-guide (UG) and show its utility and safety in a physical medicine and rehabilitation consulting room.

Methods: We present three consecutive patients with a chronic pelvic pain difficult to manage with NSAIDS or analgesics. All this patientes suffered more than six months of burning and lancinating pelvic pain that get worse with trunk movements and when the patient is seated or walking. Due to symthoms and physical examination, the patients were diagnosed of iliohypogastric neuropathy and we decided to perform an ultrasound guided iliohypogastric nerve block in all cases.

Results: The VAS punctuation were improved after each nerve blockade in three patients at the finally of follow-up, decreasing VAS 3.5 points (P50) in compare with before start the treatment. Any secondary effects were reported.

Discussion: There is little literature concerning this procedure which shows, such our study, that in blockades were used a local anesthe-
1 Hospital Universitario Virgen del Rocío, C/ Manuel Siurot SN, Seville, Seville 41013, Spain.

\section{Contact information:}

Marta Linares Gago.

झ marta.linaresgago@gmail.com 
tic and the nerve is located with ultrasound-guide. The results show partial improvement of the symptoms after the treatment.

Although our sample is small and the follow up is short, we continue the study with the aim of improving the technique and provide statistically significant results.

Conclusion: The iliohypogastric nerve block is easily repoducible in the clinical setting and offers an advancement for patients with pelvic pain, that could be an initial option of treatment to improve the pain and delay other treatment as surgery.

\section{Keywords}

Iliohypogastric Neuropathy; Pelvic Pain; Rehabilitation Treatment; Ultrasound-Guide; Nerve Block.

\section{Introduction}

Chronic Pelvic Pain is located at the lower abdomen, the pelvis or intrapelvic structures and it persists for at least six months of continuous or intermittent pain and not associated exclusively to uro-gynecological pathology [1]. There are multiple etiologies (genitourinary, gynecological, gastrointestinal, musculoskeletal, neurological, etc.) [2] and is a real diagnostic challenge for the specialist in the treatment of pain.

The most frequency causes of neuropathic Chronic Pelvic Pain are secondary to peripheral entrapment Ilioinguinal, iliohypogastric and genitofemoral nerves within stitches or scarring after abdominal surgery [3, 4]. In 2002, Deysine [5] published the differents possibilities of compressive neuropathies of the high lumbar plexus roots in the absence of surgical history and as a result of compression thereof under the inguinal ligament or muscle skeletal elements in its course.

The iliohypogastric is a purely sensory nerve derived from the first lumbar roots and enervates the skin and muscles of the lower abdominal wall as well as the skin of the proximal hip and thigh. The nerve injury may be caused by entrapment by sutures at the lateral poles of transverse fascial incisions in differents kinds of surgeries and direct or indirect traumas [6].
Examination may reveal trigger points, which cause lancinating pain when touched. If nerve block produces complete or substantial decrease in pain, neurectomy should be considered [6].

\section{Aim and Objetives}

The purpose of the study is to present the technique description of the iliohypogastric nerve block with ultrasound-guide and show its utility and safety in a Physical Medicine and Rehabilitation consulting room.

\section{Case Description}

We present three different cases:

\section{Case 1}

The first one is a 36 year old sportman without important personal backgrounds who refers low back pain eight years before, treated in 2007 by ozone therapy because of a spinal disc herniation found in a Magnetic Resonance Imaging (MRI) (L4-L5 and L5-S1). He referred an important improvement until seven months ago, when a different pain appeared.

He referred an abdominal irradiated pain that started from eleventh left rib to genital zone, with ocasional irradiation to waist region. The pain improved with the resting and it got worse when he 
was seated, load weight or makes physical activities and with the maximum hip flexion with digital presion in ipsilateral inferior abdominal region. Pain was scored in 6 points in the Visual Analogue Scale (VAS).

\section{Case 2}

The second case is a 58 years old woman with two abdominal surgeries because of a suprarrenal tumor. She referred a right superficial abdominal pain since the first one surgery, treated by intramuscular botulinum toxin in external abdominal oblique muscle and transdermal lidocaine patch by the Pain Clinic with a partial improvement. However she reported to have a located pain in the right iliac fossa, with a VAS score of 8 points and DN4 (neuropatic pain questionnaire) score of 5 points over 10. The pain was continuos and oppressive, it improved with the resting and it got worse when she was sitting down, picked weight and did physical activities.

Pain got worse with the digital presion and with pasive and active hip flexion.

Abdominopelvic Tomography Computerized (TC) only showed surgery effects.

\section{Case 3}

The last case is a 55 years old man without personal backgrounds, who referred chronic pelvic pain since two years ago despite. He was treated with second step drugs, without improvement.

The pain was located in right iliac crest and anterior superior iliac spine which was irradiated to inguinal ligament area and suprapubic region. The pain was continuous, lancinating and with burning, acompanied by hyperalgesia in hypogastric region and pubis.

Pain was of 8 points in VAS and of 6 points in DN4. It got worse when he was walking and with the extension or rotation of the trunk.

To the exploration the patient presented dorsolumbar joint pain with the extension or rotation of the trunk.
The MRI only showed a left posterolateral disc herniation L5-S1.

In all cases the neurological and motor examination of the lower limbs were normal and no red flags were found. So a diagnosis of iliohypogastric neuropathy was given and, after the patients accepted the performance of the technique and signed informed consent, we decided to do a iliohypogastric nerve blockade.

\section{Technique description}

We used a linear transducer at a frequency of 10 $\mathrm{MHz}$ to identify the inguinal ligament, near the anterior superior spine of the ilium [7]. Then, we located the iliohypogastric nerve situated between the internal abdominal oblique and the transversus abdominis muscles (figure 1, 2 and 3).

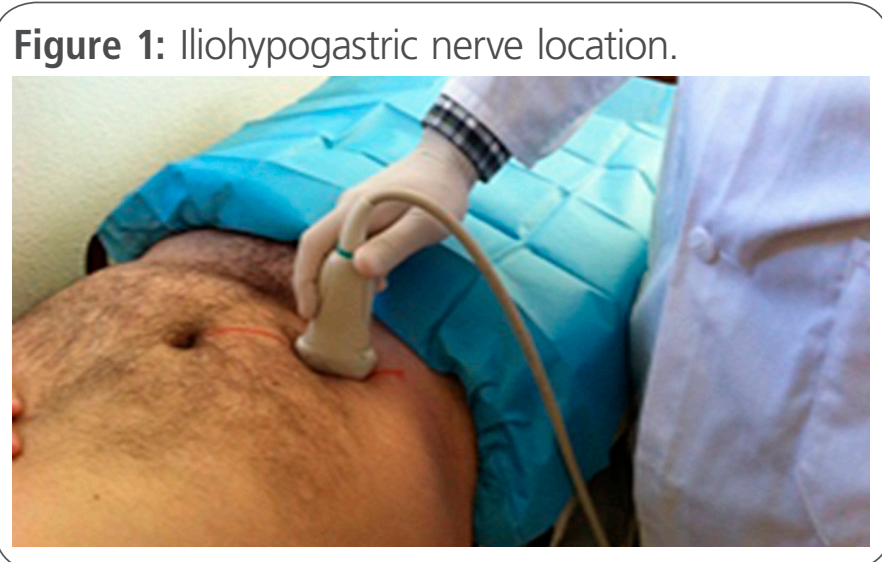

Figure 2: ultrasound-guide location.

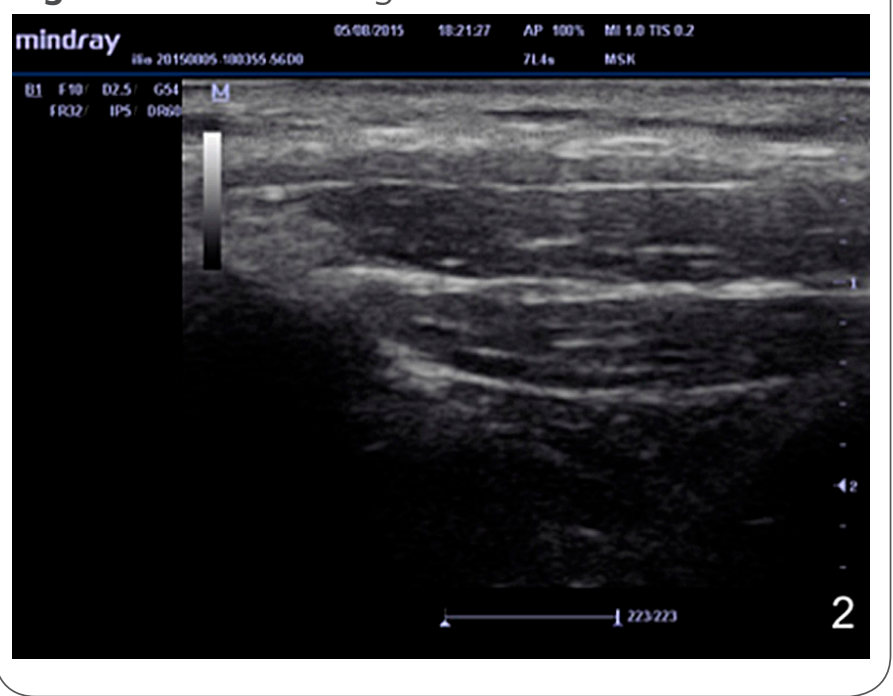


Figure 3: ASIS: anterior superior ilium spine, IAB: internal abdominal oblique, TA: transversus abdominis, ILN: Iliohypgastric nerve (the yellow one).

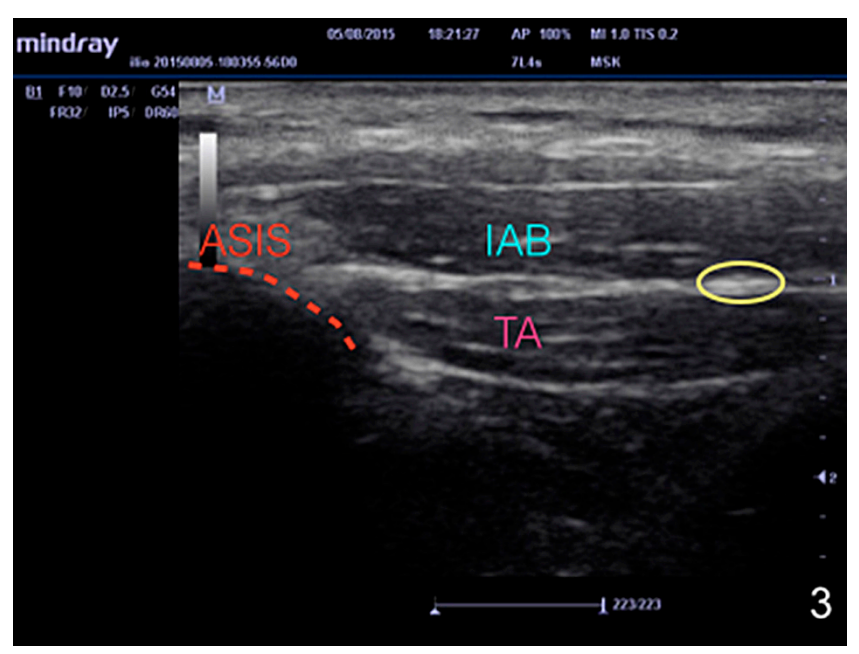

Figure 4: The grey line marks the needle trajectory. The end of this one are in the perineurial recess. In this place must be done the nerve block.

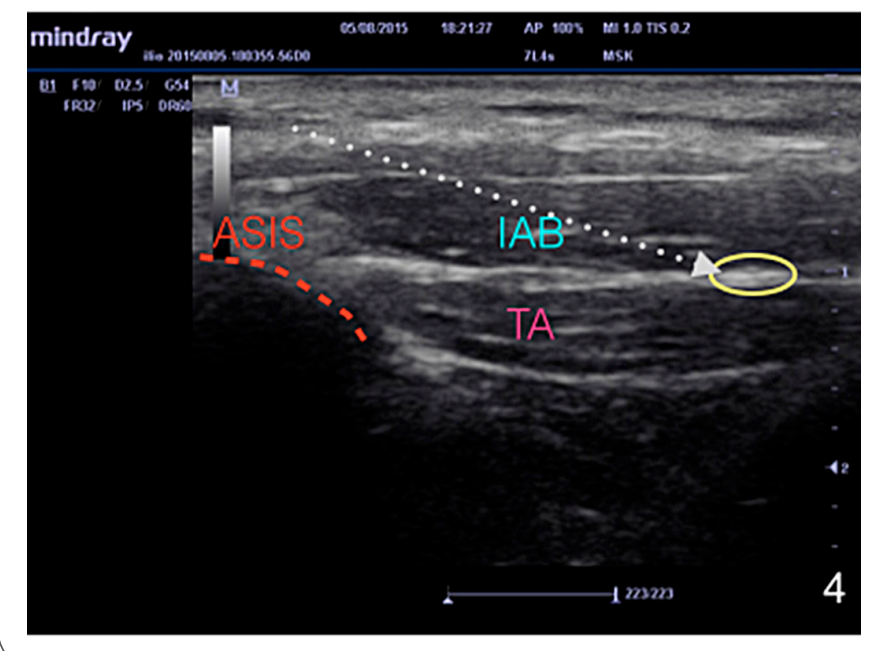

Once identified the nerve, we proceeded to perform the nerve block with $5 \mathrm{ml}$ of bupivacaine using a 21 gauge needle $(4 \mathrm{~cm})$. We introduced the needle in the large axis to reach the perineurial nerve area (figure 4).

\section{Results}

Regarding the VAS score prior to infiltration, P50 was of 8 points (range 6-8). Immediately after the infiltration, all patients showed a decrease of the pain.

One month later, the VAS P50 was of 3.5 (range 0-7). We decided to do a second block in two patients because the other one had got a pain disappearance. One month after doing the second block, VAS P50 was 4.5 (range 4.5-5) in this two patients. (Figure 5)

The response to treatment was not associated with either sex or with BMI or the type of oral treatment done. There was no relation to the time of onset of symptoms or the fact of having previous abdominal surgery. Any secondary effect were reported after the follow up.

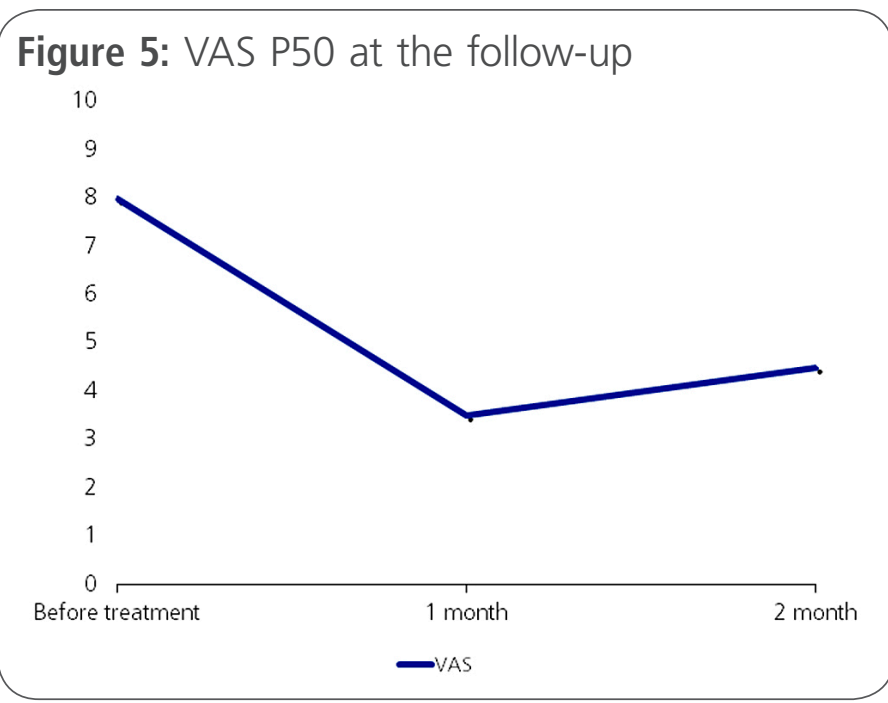

\section{Discussion}

We have found in the literature review only one study about the abdominal nerve blockade for the treatment of the pelvic pain [8], being in that case the ilioinguinal nerve block. Such as our study, a local anesthetic were used to do the blockades and the nerve was located with ultrasound-guide.

About the pain improvement, in Acevedo's study sample, of 35 patients that were made the ilioin- 
guinal blockade the symptoms improved after the intervention in 7 cases. In our study, all of patients had an important improvement of the pain after the blockades, but one of them showed a little aggravation of the symptoms at the final of the follow-up (Table 1).

We are conscious that the sample size is small and the follow-up short. At the present we continue working in the treatment of patients with pelvic pain symptoms with the aim of improve the technique and show a statistically significant outcomes.

Table 1. VAS punctuation at the follow-up.

\begin{tabular}{|l|c|c|c|}
\hline $\begin{array}{c}\text { Patient } \\
(\text { P) }\end{array}$ & $\begin{array}{c}\text { VAS } \\
\text { before } \\
\text { treatment }\end{array}$ & $\begin{array}{c}\text { VAS } \\
\text { 1 month }\end{array}$ & $\begin{array}{c}\text { VAS } \\
\text { 2 month }\end{array}$ \\
\hline P1 & 8 & 0 & - \\
\hline P2 & 8 & 7 & 5 \\
\hline P3 & 6 & 3.5 & 4.5 \\
\hline
\end{tabular}

\section{Conclusion}

The iliohypogastric nerve block is easily repoducible in the clinical setting and offers an advancement for patients with pelvic pain and could be an initial option of treatment, improving the pain and delaying other treatment as surgery.

In addition, ultrasound-guiadance can facilitate iliohypogastric nerve blocake for diagnostic and therapeutic purposes and may be particularly beneficial with patients with challenging surface anatomic landmarks.

Although there is little literature concerning this procedure, the references presented suggest that anesthetic blockade of abdominal nerves could be a therapeutic option in the management of patients with chronic pelvic pain of neuropathic nature and poor response to conventional analgesia.

\section{References}

1. J. Moore. Causes of chronic pelvic pain. Bailliere's Clinical Obstetric and gynaecology. 2000. vol 14, n³, 398-402.

2. Mardsen AJ. Ilioinguinal hernia: a three year review of two thousand cases. Br J Surg. 1962 Jan; 49: 384-94.

3. Waldman S, Rini D, Phelps T. Neuralgia Genitofemoral. Atlas de síndromes doloroso frecuentes. Elsevier España, 2003.

4. Nahabedian MY. Outcome of operative management of nerve injuries in the ilioinguinal region. J Am Coll Surg. 1997 Mar; 184(3): 265-8

5. Deysine M, Desyne GR and Reed WP. Groin Pain in the abstence of hernia: a new syndrome. Hernia. 2002 Jul; 6(2): 64-7.

6. Jeremaine EG. Nerve injury associated with pelvic surgery. In: UpToDate, Waltham, MA. (Accessed on Dec 5, 2013.)

7. Philip WP. Ultrasound-Guided Interventional Procedures for Patients with Chronic Pelvic Pain. A description of Techniques and Review of Literature. Pain Physician. 2008; 11: 215-24.

8. Acevedo A et al. Chronic inguinal pain syndrome. Rev. Chilena de Cirugía. Vol 61 - n³, Junio 2009; pág. 249-255.

\section{Comment on this article:}

\section{f) (B) in $8+\mathbf{S}$ ?}

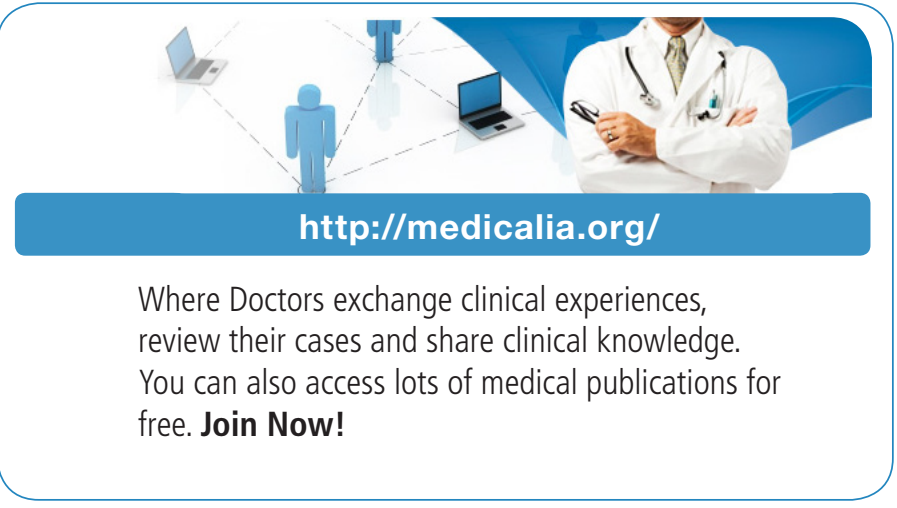

Publish with iMedPub

\section{http://www.imed.pub}

International Archives of Medicine is an open access journal publishing articles encompassing all aspects of medical science and clinical practice. IAM is considered a megajournal with independent sections on all areas of medicine. IAM is a really international journal with authors and board members from all around the world. The journal is widely indexed and classified Q1 in category Medicine. 